\title{
Mesure humaine et démesure technologique
}

Une iconologie de la « crise de civilisation » dans les années 1930

Human measure and technological sublime. An iconology of the "civilisation

crisis" in the 1930s

\section{Max Bonhomme}

\section{(2) OpenEdition}

1 Journals

\section{Édition électronique}

URL : http://journals.openedition.org/artefact/3181

DOI : $10.4000 /$ artefact.3181

ISSN : 2606-9245

Éditeur :

Association Artefact. Techniques histoire et sciences humaines, Presses universitaires du Midi

\section{Édition imprimée}

Date de publication : 15 mars 2019

Pagination : 175-191

ISBN : 978-2-8107-0623-5

ISSN : 2273-0753

\section{Référence électronique}

Max Bonhomme, " Mesure humaine et démesure technologique ", Artefact [En ligne], 9 | 2018, mis en ligne le 04 mars 2020, consulté le 27 novembre 2020. URL : http://journals.openedition.org/artefact/ 3181 ; DOI : https://doi.org/10.4000/artefact.3181

\section{(ब) $\odot \Theta$}

Artefact, Techniques, histoire et sciences humaines est mise à disposition selon les termes de la Licence Creative Commons Attribution - Pas d'Utilisation Commerciale - Pas de Modification 4.0 International. 


\title{
Mesure humaine et démesure technologique
}

\author{
Une iconologie de la « crise de civilisation » dans les \\ années 1930
}

Max Bonhomme

\section{Résumé}

Les outils de l'iconologie, appliqués à la presse illustrée des années 1930, permettent de mettre en évidence le sens politique et idéologique de l'imagerie du déclin civilisationnel, omniprésente dans ces années. L'inquiétude grandissante face au développement du « machinisme » tend à se formuler visuellement par des rapports d'échelle : on opposera alors le gigantisme des machines à la petitesse de l'homme, menacé d'être dépassé par ses propres réalisations. L'article retrace la migration de certains motifs, comme celui d'un homme pris dans d'immenses engrenages, qui cheminent de l'iconographie militante vers la culture de masse. Différentes visions du progrès technique sont à distinguer, en fonction des contextes culturels et nationaux : ainsi la démesure technologique, qui caractériserait à la fois les États-Unis et l'URSS, définit en creux la situation de la France à cette époque, volontiers présentée comme le «pays de la mesure ».

\section{Mots-clés}

France, crise (années 1930), iconologie, technologie, machinisme, histoire de la presse, photomontage

95 Max Bonhomme, « Mesure humaine et démesure technologique. Une iconologie de la “crise de civilisation" dans les années 1930 », Artefact, 9, 2018, p. 175-191. 


\section{Human measure and technological sublime. An iconology of the "civilisation crisis" in the 1930s}

\section{Abstract}

The tools of iconology, applied to the illustrated press of the 1930s, highlight the political and ideological meaning of the imagery of civilizational decline, which is pervasive during these years. The growing anxiety about the development of mechanization is formulated visually in terms of scale: the gigantism of machines is opposed to the smallness of man, threatened to be overwhelmed by his own achievements. The article traces the migration of some patterns, such as that of a man caught in huge gears, that circulate from militant iconography to mass culture. Diverging visions of technical progress are to be distinguished, depending on cultural and national contexts: thus, the vision of a technological sublime, shared by both Soviet and American imagery, is to be understood as a counter-model in French interwar culture, which promotes France as "the country of balance".

\section{Keywords}

France, crisis (1930s), iconology, technology, mechanization, history of the press, photomontage 
$\mathrm{P}$ ar rapport à l'enthousiasme technophile qui caractérise, globalement, les années 1920, la culture visuelle des années 1930 voit l'émergence d'une imagerie du déclin civilisationnel, nourrie d'images à portée allégorique, dont nous nous proposons d'analyser ici la rhétorique. L'hypothèse de base de cette enquête consiste à supposer que l'angoisse technologique se formule visuellement par des rapports d'échelle. À l'idéal humaniste de l'homme compris comme «la mesure de toutes choses ", s'oppose, dans les années 1930, une image de l'homme dépassé et menacé par ses propres réalisations techniques. Certes, ce type d'images n'est pas majoritaire dans la culture de masse. En pleine crise, la presse illustrée continue de glorifier les progrès technologiques accomplis par les pays occidentaux, alors que se développe une nouvelle culture de la consommation épousant la modernité technique (automobiles, aviation, TSF, prémices de la télévision). Le développement de la presse illustrée par la photographie, en tant que principal média de masse, participe pleinement de cette culture moderniste tout en se faisant l'écho, non sans paradoxe, des critiques de plus en plus vives à l'égard de la civilisation industrielle et des dangers de la technique.

En mars 1933, l'hebdomadaire illustré $V u$ publie un numéro spécial consacré aux conséquences sociales du "machinisme » et aux menaces qu'il représente pour la civilisation ${ }^{1}$. Publié au cœur de la crise économique, qui touche la France avec quelques années de retard par rapport aux ÉtatsUnis, le magazine se fait l'écho d'un débat qui agite le monde intellectuel français depuis la fin des années 1920 et que Georges Duhamel a désigné sous le nom de "querelle du machinisme ${ }^{2}$ ». Un photomontage de Marcel Ichac illustre la couverture (Fig. I, cahier couleur), qui se présente comme une allégorie de la crise de civilisation telle qu'elle se cristallise alors dans les représentations. Ce motif d'un homme prisonnier d'engrenages gigantesques, dont le sens est explicité par le titre du numéro, "Fin d'une civilisation ", s'inscrit dans une vaste série d'images similaires, parmi lesquelles une célèbre séquence du film de Charlie Chaplin, Les Temps modernes (1936).

1. $V u, \mathrm{n}^{\circ} 259,1^{\text {er }}$ mars 1933. À propos de $V u$ et du parcours de son directeur, Lucien Vogel, voir Sophie Kurkdjian, Lucien Vogel et Michel de Brunhoff, parcours croisés de deux éditeurs de presse illustrée au XXe siècle, Bayonne, Institut universitaire Varenne, 2014.

2. Georges Duhamel, "Sur la querelle du machinisme ", Revue de Paris, XL, 15 avril 1933, p. 721 752. Voir Michel Raimond, Éloge et critique de la modernité, Paris, PUF, 2000, chap. 5, p. 92-117. 
Doit-on considérer ces images de la " crise du progrès " comme des allégories politiques ? Il est tentant, en effet, d'y voir l'équivalent visuel de la rhétorique du déclin très prisée des pamphlétaires d'extrême droite ${ }^{3}$. Cependant, l'usage politique de ces images révèle des positions idéologiques diverses et parfois antithétiques. Pour sortir de cette contradiction, il est nécessaire de revenir à la fois sur la diversité des discours anti-machinistes dans les années 1930 et de mettre en évidence le fonctionnement propre de l'imagerie en question. Il s'agit alors d'identifier la cohérence d'un corpus d'images à la fois visuelles et verbales, caractérisées par l'emploi des mêmes figures de style. Tout en nous situant dans le cadre d'analyse des études visuelles, notre étude se situe dans la continuité d'une approche iconologique commencée par Aby Warburg et Erwin Panofsky, considérant les images, quel que soit leur support, comme des allégories sociales. Plus récemment William J. T. Mitchell a insisté sur le rôle joué par les images dans « la construction visuelle du champ social », ouvrant la voie à une approche intermédiale considérant non seulement ce que l'on dit sur les images mais aussi par les images ${ }^{4}$. Outre donc les formules rhétoriques spécifiquement visuelles souvent mises en avant à propos de l'imagerie publicitaire ou de propagande $e^{5}$, il faudra aussi prendre en compte (dans le cas d'images de presse, celles qui nous occuperont ici $\left.{ }^{6}\right)$ à la fois le contexte de publication et la chaîne "intericonique ${ }^{7}$ " dans laquelle s'inscrivent les images. La notion d'imagerie narrative, proposée par André Gunthert, décrit bien ces processus de mise en récit, tout en insistant sur la dynamique d'imitation et de reprise propre au fonctionnement des industries culturelles ${ }^{8}$.

3. Juliette Rennes, "L'argument de la décadence dans les pamphlets d'extrême droite des années $1930 "$ Mots, vol. 58, n 1, 1999, p. 152-164.

4. William John Thomas Mrtchell, Iconologie : image, texte, idéologie, trad. Maxime Boidy et Stéphane Roth, Paris, Les Prairies ordinaires, 2018.

5. Carlo Ginzburg, Peur, révérence, terreur : quatre essais d'iconographie politique, Dijon, Presses du réel, 2013.

6. L'analyse pourrait être poursuivie en prenant en compte d'autres supports, en particulier le cinéma.

7. Mathilde Arrivé, «L'intelligence des images : l'intericonicité, enjeux et méthodes », E-rea, 13/1, 2015, en ligne : https://journals.openedition.org/erea/4620 (consulté le 31 octobre 2018).

8. "À la question : que font les images ? on peut répondre : elles produisent d'autres images. Cette productivité caractéristique permet de distinguer entre une iconographie, groupe d'images isolé à partir d'un critère quelconque, et une imagerie définie par son succès public, qui présente des traits de cohérence interne, mais aussi une dynamique évolutive... " : André Gunthert, "Comment 
Ainsi, le tropisme commun de cette imagerie du déclin civilisationnel qui se développe autour de 1930 pourrait se résumer à quelques éléments récurrents : premièrement, l'image des engrenages comme métonymie pour désigner soit le travail industriel, soit le travail en général, soit un phénomène de mouvement autonome et incontrôlable ; deuxièmement une dépersonnalisation de la figure humaine réduite à une silhouette dont on distingue mal l'identité ${ }^{\text {; }}$ troisièmement, la disproportion entre l'homme et la machine, qui tend à maximiser le contraste par la rupture d'échelle.

\section{Les critiques $\mathrm{du}$ " machinisme » en France et en Europe}

La couverture de $V u$ ne peut se comprendre en dehors du contexte idéologique de la France autour de 1930, marqué par un vif débat sur les mérites et les dangers du "machinisme ». Si l'intensité de la polémique s'accentue après 1931, lorsque se font sentir les effets de la crise économique, il s'agit en fait d'un topos littéraire dont les racines remontent au romantisme ${ }^{10}$. Les oppositions à la mécanisation, émanant à la fois du monde intellectuel et de résistances ouvrières, avaient été jusqu'alors plutôt mesurées dans le contexte français, par rapport à la situation anglaise par exemple ${ }^{11}$. Après les ravages de la Première Guerre mondiale, l'enthousiasme pour le progrès technique est déjà fortement altéré : on conçoit désormais clairement que " la machine " peut s'avérer destructrice et que la guerre industrielle peut prendre des formes particulièrement inhumaines. Cette mémoire de la guerre pèse fortement sur les auteurs qui, dans l'entre-deux-guerres, se placent du côté des contempteurs du " machinisme».

Georges Duhamel, l'une des principales figures de ce débat, rappelle en effet l'importance qu'a eue pour lui l'expérience de la guerre dans la prise

\footnotetext{
lisons-nous les images ? Les imageries narratives ", in Gil Bartholeyns (dir.), Politiques visuelles, Dijon, Presses du réel, 2016, p. 219-234, p. 223.

9. Nous faisons l'hypothèse que c'est l'humanité dans son ensemble qui est ainsi désignée, et non pas seulement le travailleur industriel. Dans certains cas la référence à la chaîne de montage est plus explicite, et cela désignerait alors l'anonymat et l'aliénation que ferait subir la rationalisation scientifique du travail à l'individu.

10. Serge Audier, La société écologique et ses ennemis, Paris, La Découverte, 2017.

11. François Jarrige, Techno-critiques. Du refus des machines à la contestation des technosciences, Paris, La Découverte, 2014.
} 
de conscience du caractère potentiellement destructeur de la technologie. En 1930, Duhamel publie un récit de voyage au ton pamphlétaire sous le titre Scènes de la vie future, qui connaîtra un succès considérable. Dans ce texte qui relate un voyage de l'écrivain aux États-Unis à la fin de l'année 1929, l'écrivain critique le supposé matérialisme de la civilisation américaine, qu'il détecte à la fois dans la production industrielle, dans la vie quotidienne de plus en plus mécanisée, ainsi que dans les productions culturelles (le cinéma, la musique enregistrée). Dans ce que Duhamel considère comme un excès de rationalisme, sont visées à la fois les transformations du travail dues à la mécanisation, qu'il oppose au savoir-faire qualifié et au travail manuel, et les transformations de la culture, devenue culture de masse, en particulier le cinéma dont il attaque le rythme selon lui effréné et peu propice à la réflexion. Scènes de la vie future est beaucoup commenté et donne lieu à un débat qui anime toute la presse d'opinion autour de $1930^{12}$. Nombreux sont les ouvrages contemporains qui multiplient ces arguments contre le machinisme en développant une rhétorique de la démesure ${ }^{13}$.

Pour Georges Duhamel comme pour la plupart des auteurs français prenant part à la « querelle ", les États-Unis constituent l'emblème de la civilisation machiniste moderne qui se caractériserait par sa démesure. Le gratte-ciel est l'un des symboles de cette démesure technologique, dont Jean-Louis Cohen a bien montré à quel point il a marqué, souvent par la négative, l'imaginaire architectural français ${ }^{14}$. C'est dans cette notion de démesure que se nouent enjeux politiques et esthétiques, car la figure-repoussoir des États-Unis (mais aussi de son miroir oriental, l'URSS), représente pour ces auteurs un danger à l'esprit de mesure considéré comme spécifiquement

12. Anne-Marie Duranton-Crabol, "De l'anti-américanisme en France vers 1930 : la réception des Scènes de la vie future ", Revue d'histoire moderne et contemporaine, $\mathrm{n}^{\circ}$ 48-1, 2001, p. 120-137. Voir aussi Gérard de Catalogne, Dialogue entre deux mondes : enquête, Paris, Alexis Redier, 1931. Concernant les conséquences de l'américanisme dans le domaine de l'architecture, voir Jean-Louis CoHen (dir.), Scènes de la vie future : l'architecture européenne et la tentation de l'Amérique, 18931960, Paris/Montréal, Flammarion/Centre canadien d'architecture, 1995.

13. Jean-Léopold Duplan, Sa majesté la machine, Paris, Payot, 1930 ; Gina Lombroso, La rançon du machinisme, Paris, Payot, 1931 ; Louis Hoyack, Où va le machinisme ?, Paris, Marcel Rivière, 1931 ; Henri Bergson, Les deux sources de la morale et de la religion, Paris, Félix Alcan, 1932 ; Henri Daniel-Rops, Le monde sans âme, Paris, Plon, 1932 ; Nicolas Berdiaev, L'Homme et la Machine, Paris, Éditions Je sers, 1933.

14. J.-L. Cohen (dir.), Scènes de la vie future, op. cit. 
français. Chez Duhamel, Amérique et gigantisme sont indissociables, ne serait-ce que par l'échelle même du territoire :

Ses cités inhumaines, le peuple américain les a dressées sur un sol qui, jamais, n'invite à la modération. Lacs, vallées, rivières, forêts, plaines, tout est démesuré, rien ne semble fait pour incliner l'homme vers une pensée d'harmonie. Tout y est trop grand. Tout y décourage Apollon et Minerve ${ }^{15}$.

Contrairement donc aux critiques de la mécanisation émises depuis le $\mathrm{XIX}^{\mathrm{e}}$ siècle, portant essentiellement sur la nature du travail industriel et parfois sur les conséquences environnementales ${ }^{16}$, les discours du déclin autour de 1930 vont jusqu'à anticiper la destruction de l'humanité par elle-même. La science-fiction fera d'ailleurs sienne, avec une postérité considérable, la fiction du soulèvement des machines ${ }^{17}$, résultant du péché originel prométhéen. Ce discours à tonalité beaucoup plus tragique (comparé notamment aux satires voltairiennes de Duhamel), a été notamment popularisé par Oswald Spengler en Allemagne. La réception de Spengler en France fut cependant des plus limitée, ce qui n'a rien d'étonnant étant donné le caractère explicitement francophobe du Déclin de l'Occident (1918$1922)^{18}$. La technologie a un statut ambivalent chez Spengler : considérée comme le propre de la civilisation occidentale dans son rapport «faustien » à la nature, le développement exponentiel qu'elle a connu dans la période récente témoignerait selon lui d'une domination néfaste du matérialisme, symptôme du déclin civilisationnel qu'il condamne ${ }^{19}$. Mais c'est surtout chez Spengler que l'on trouve formulée de manière la plus explicite l'idée

15. Georges Duhamel, Scènes de la vie future [1930], Paris, Mille et une nuits, 2003, p. 81.

16. Jean-Baptiste Fressoz, L'apocalypse joyeuse : une histoire du risque technologique, Paris, Seuil, 2012.

17. Le mythe du soulèvement des machines remonte au $\mathrm{XIX}^{\mathrm{e}}$ siècle, en particulier à l'œuvre de Samuel Butler (1835-1902) qui fait l'objet d'un regain d'intérêt dans les années 1930 : voir JeanBaptiste ForT, "Les idées de Samuel Butler ", Revue Philosophique de la France et de l'Étranger, t. 122, n $^{\circ}$ 9/10, sept-oct.1936, p. 215-239.

18. Oswald Spengler, Le Déclin de l'Occident : esquisse d'une morphologie de l'histoire universelle [1918-1922], trad. M. Tazerout, Paris, Gallimard, 1931 [1 ${ }^{\text {re }}$ partie] et 1933 [2 $2^{\mathrm{e}}$ partie] pour la première édition française.

19. "La créature se dresse contre son créateur. De la même façon que le microcosme Homme se révolta un jour contre la Nature, ainsi fait aujourd'hui le microcosme Machine se révoltant contre l'Homme Nordique. Le maître du Monde est en train de devenir l'esclave de la Machine qui le force [...] à en passer par où elle veut. Abattu, le triomphateur est traîné à mort par le char. » Oswald Spengler, L'Homme et la technique [1931], trad. A. Petroswky, Paris, Gallimard, 1958, p. 155-156. 
d'une démesure technologique, à la fois fascinante et terrifiante, dans des termes qui s'inscrivent l'esthétique du sublime. La technique moderne témoignerait en effet d'un élan "vers des lointains sans limite ${ }^{20}$ »:

C $C$ C'est pour cela que le mot prononcé est envoyé instantanément au-delà des mers ; c'est pour cela qu'on assiste à cette ambition des records et des dimensions, géants hangars pour des machines géantes, bateaux et ponts effrayants, constructions de gratte-ciel ; forces fabuleuses qui obéissent, en un point pressé, à la main d'un enfant ; usines d'acier et de verre qui tanguent, tremblent, gémissent, et dans lesquelles le minuscule homunculus circule en maître absolu et sent finalement la nature au-dessous de lui ${ }^{21}$.

Lucien Febvre a justement remarqué à quel point la théorie de l'histoire chez Spengler se nourrit des angoisses de l'Allemagne des années 1920 et combien la rhétorique du déclin civilisationnel nourrit une joie malsaine (Schadenfreude) qui consisterait à anticiper sa propre ruine :

Spengler tenait boutique, en ces années 20, des denrées alors les plus convoitées disons, un certain pathétique, un antiintellectualisme résolu, la notion héroïque du destin, l'antiesthéticisme, le frisson de la créature humaine devant la majesté, l'ample majesté de l'Histoire ${ }^{22}$.

En France, certains auteurs qualifiés de «non-conformistes », qui partagent pour la plupart un rejet du parlementarisme et du libéralisme, rejoignent certaines thèses spengleriennes concernant le rôle de la technique dans le « déclin » civilisationnel, considéré comme une victoire néfaste du matérialisme sur «l'esprit ${ }^{23}$ ». Il serait cependant inexact d'attribuer uniquement

Voir aussi Éric Michaud, "Figures nazies de Prométhée, de l'"homme faustien” de Spengler, au "Travailleur" de Jünger ", Communications, n 78,2005 , p. 163-173.

20. Oswald SPEngLer, Le déclin de l'Occident, esquisse d'une morphologie de l'histoire universelle, t. II [1922], trad. M. Tazerout, Paris, Gallimard, 1948, p. 462.

21. Ibid., p. 463.

22. Lucien Febvre, "De Spengler à Toynbee, quelques philosophies opportunistes de l'histoire ", Revue de métaphysique et de morale, $\mathrm{n}^{\circ}$ 4, 1936, p. 573-692, p. 579.

23. Jean-Louis LoubET DEL BAYLE, Les non-conformistes des années 30 : une tentative de renouvellement de la pensée politique française, Paris, Seuil, 1987 ; Olivier DARD, Le rendez-vous manqué des relèves des années trente, Paris, Presses universitaires de Ffrance, 2002. Dès les années 1920 en réalité, la mécanisation se trouve associée chez certains auteurs conservateurs à une forme de perversion contre-nature : « La machine est un artisan automate par quoi l'homme, leurré et épuisé par l'effort même de cet enfantement, prétend se faire remplacer. Mais il est trahi. Il se chagrine et s'abîme dans 
ces critiques de la modernité à une pensée conservatrice, non seulement parce que les dictatures ont souvent mis en avant une réconciliation de façade entre ancrage dans la tradition et technologie prométhéenne ${ }^{24}$, mais aussi parce qu'il a existé des courants anarchistes et socialistes qui mettaient également en garde - avec d'autres arguments, il est vrai - contre les possibles dangers du développement technologique (avec des positions qui ont contribué à former le concept moderne d'écologie politique ou de société écologique ${ }^{25}$ ).

\section{De l'ouvrier crucifié à l'ouvrier- monument}

Du point de vue iconographique, la couverture de $V u$ appelle une autre généalogie, témoignant de certaines survivances mythologiques dans l'iconographie politique du $\mathrm{xx}^{\mathrm{e}}$ siècle. En effet, le photomontage de Marcel Ichac convoque des références visuelles dont les connotations négatives se rattachent à l'iconographie de la torture (le supplice de la roue) et à la représentation des enfers voire au supplice d'Ixion (en particulier l'arrière-plan de l'image qui évoque des flammes). En réalité, cette association d'idées entre travail mécanisé et instrument de torture est apparue au sein de l'iconographie militante, anarchiste notamment. Ainsi, en 1906, le peintre František Kupka publie une lithographie (Fig. II, cahier couleur) représentant un ouvrier crucifié sur une roue dentée, dans le journal anarchiste Les Temps nouveaux ${ }^{26}$.

Ce type iconographique fonctionne comme une analogie visuelle entre travail et torture, et il est possible d'y voir une référence à l'étymologie supposée du mot " travail », que l'on a parfois fait dériver du mot romain tripalium, un instrument de torture utilisé dans l'Antiquité pour punir les esclaves rebelles. Bien que cette étymologie ait été remise en cause par les

\footnotetext{
ses vaines machinations comme dans les substitutions de la vie sexuelle. [...] Car il ne peut être question de la répudier, [...] il faut conjurer la déviation, l'inversion. " Pierre Drieu La Rochelle, Mesure de la France, Paris, Grasset, 1922, p. 111-112.

24. Jeffrey Herf, Reactionary Modernism: Technology, Culture, and Politics in Weimar and the Third Reich, Cambridge, Cambridge University Press, 1984.

25. S. Audier, La société écologique et ses ennemis, op. cit.

26. Les Temps nouveaux, $\mathrm{n}^{\circ} 32,8$ décembre 1906, p. 8.
} 
linguistes ${ }^{27}$, il est probable qu' elle ait nourri les analogies que l'on observe dans l'iconographie politique de cette époque ${ }^{28}$. Les reprises de cette métaphore visuelle sont particulièrement nombreuses dans les années 1930, en relation avec les critiques de l'organisation "scientifique " du travail (taylorisme). L'illustrateur communiste américain Hugo Gellert publie par exemple en 1934 une interprétation en images du Capital de Karl Marx, dans laquelle une ouvrière et un ouvrier sont représentés torturés sur d'immenses engrenages (illustration du chapitre 15, "Machinisme et grande industrie »). La métaphore joue sur deux plans : l'ouvrier est réduit à un simple rouage dans la machine industrielle, mais il est aussi sacrifié (tel le Christ crucifié) sur l'autel du profit capitaliste.

À cette imagerie négative du travail industriel en régime capitaliste répondent les stratégies visuelles affirmatives de la propagande communiste, qui tendent à inverser le rapport d'échelle entre l'homme et la machine. Si le motif de l'ouvrier-crucifié dans l'iconographie socialiste renvoyait à la prégnance du concept d'aliénation chez le jeune Marx, ce motif fait place à une iconographie technophile à l'heure du communisme soviétique. De l'ouvrier dominé par l'élément mécanique, on passe alors à l'ouvrier-monument, surplombant désormais les moyens de production dont il serait devenu maître. Un photomontage de John Heartfield, en couverture du magazine communiste allemand Arbeiter Illustrierte Zeitung (Fig. III, cahier couleur) fonctionne ainsi sur le principe de l'hyperbole visuelle, dont le sens est orienté par les rapports d'échelle. Le visage d'un ouvrier soviétique occupe les deux tiers de l'image, surmontant des bâtiments industriels qui recouvrent les bras de l'homme qui pourraient presque évoquer les membres d'un hybride homme-machine. S'il fallait encore lever l'ambiguïté, le titre de l'image vient en fixer la signification : «Un homme nouveau - Maître d'un monde nouveau ».

Le communisme, en effet, a pour trait caractéristique une vision émancipatrice de la technologie, dans la mesure où celle-ci serait aux mains des travailleurs et non des capitalistes. On sait par exemple avec quel enthousiasme furent accueillis les principes tayloristes dans la Russie de Lénine. À l'heure de la « querelle du machinisme ", les communistes ne manquent

27. André EskéNAZI, «L'étymologie de Travail», Romania, vol. 126, n 503, 2008, p. 296-372.

28. Christoph Bertsch, "Der grekreuzigte Arbeiter. Anmerkung zu einem vernachlässigten Bildtypus der Zwischenkriegzeit ", in Klaus Türk (dir.), Arbeit und Industrie in der bildenden Kunst, Stuttgart, Steiner, 1997, p. 40-49. 
pas de prendre part au débat, pour fustiger le caractère réactionnaire des contempteurs «bourgeois " du machinisme ${ }^{29}$. La position du PCF à cet égard est ainsi résumée par Georges Friedmann ${ }^{30}$ dans le magazine communiste Regards:

Le travail à la chaîne, en lui-même, facilite la production, la rend plus rapide, souvent plus aisée. Mais il faut pour cela (comme dit encore à propos de l'outil, Marx) que l'homme s'en serve et non pas qu'il le serve. Ce contrôle de la machine n'est possible qu'en régime socialiste où les instruments de production sont réellement exploités au bénéfice de la collectivité, comme nous le voyons en URSS ${ }^{31}$.

Il faut souligner à cet égard l'importance du magazine L'URSS en Construction $^{32}$, revue de propagande illustrée de photographies et mise en page par des artistes pour la plupart venus du constructivisme, qui contribue beaucoup à diffuser en Europe de l'Ouest l'idéal soviétique du progrès technologique, magnifié par le grand format du magazine $(30 \mathrm{x}$ $41 \mathrm{~cm}$ ) et la qualité des photographies.

Du point de vue visuel, l'un des éléments récurrents de la propagande communiste consiste à formuler une analogie entre le gigantisme de la machine et le gigantisme du travailleur lui-même. C'est le cas par exemple avec la couverture d'un ouvrage de Paul Vaillant-Couturier, édité par le Bureau d'éditions du Parti communiste français ${ }^{33}$. Le lettrage bleu du titre, "Les géants industriels", se superpose à une photographie de deux travailleurs vus en contre-plongée, ce qui accentue l'effet de monumentalisation. Le photomontage se prête particulièrement bien à ces jeux d'échelle

29. Georges Friedmann, Problèmes du machinisme en URSS et dans les pays capitalistes, Paris, Éditions Sociales Internationales, 1934 ; Georges Friedmann, « Machine et humanisme ", Europe, 15 juillet 1935 .

30. La position de Georges Friedmann va considérablement évoluer suite au pacte germano-soviétique et à l'expérience de l'occupation, qui le conduit à changer de point de vue sur la paysannerie. Le terme de "machinisme " acquiert dès lors chez Friedmann des connotations négatives. Voir François VATIN, «Machinisme, marxisme, humanisme : Georges Friedmann avant et après guerre ", Sociologie du travail, vol. 46, $\mathrm{n}^{\circ}$ 2, 2004, p. 205-223.

31. Georges Friedmann, "Machinisme ", Regards sur le monde du travail, nº 16, avril 1933, n.p. 32. Le magazine est publié dans différentes langues à partir de 1931, notamment russe, français, allemand, anglais et espagnol.

33. Paul Vaillant-Couturier, Les bâtisseurs de la vie nouvelle : neuf mois de voyage dans l'URSS du plan quinquennal. Tome 3: Les géants industriels, Paris, Bureau d'éditions, 1932. 
antinaturalistes, dans la mesure où il est fondé sur l'assemblage de fragments photographiques, parfois hétéroclites dans leurs tailles, sans nécessairement s'appuyer sur les règles de la perspective linéaire. On peut à ce propos parler de perspective signifiante ou de perspective d'importance pour désigner une image dans laquelle les objets sont représentés non en fonction de leur situation spatiale mais en fonction de leur importance symbolique, comme souvent dans la peinture médiévale. Dans les photomontages des années 1920-1930, on trouve aussi des utilisations de la perspective inversée (les lignes de fuite ne se rencontrent pas dans le fond de l'image mais à l'avant de celle-ci). Pour l'historien de l'art Devin Fore, ce renversement de perspective témoignerait d'une volonté de mettre à mal le paradigme humaniste anthropocentrique, notamment au sein des avantgardes constructivistes qui célébraient la nouvelle culture de la machine ${ }^{34}$.

\section{La machine sublimée}

Dans les États-Unis des années 1930, les représentations de l'industrie dans la presse illustrée s'appuient souvent sur une esthétique du sublime réactivée, ce que David Nye a qualifié de technological sublime ${ }^{35}$. Destiné à contrer l'idée même d'aliénation et de risque technologique, en inscrivant l'industrie moderne dans un horizon de maîtrise du territoire et de dépassement des frontières (frontier), l'esthétique du sublime technologique est indissociable d'un projet politique : maintenir le ferment de l'unité nationale en période de crise, en réinstaurant une transcendance, autrefois associée au paysage naturel sauvage et indompté (wilderness), et désormais aux œuvres de l'homme lui-même. David Nye souligne également le fait que le sublime est indissociable d'un sentiment de terreur, mêlé à de l'admiration. En principe associé aux puissances illimitées de la nature, le sublime suscité par les créations humaines (Nye donne comme exemples les ponts, les barrages, les tours) peut induire l'idée d'un danger pour l'homme lui-même, dépassé par l'échelle de ses propres réalisations.

34. Devin Fore, Realism after Modernism: The Rehumanization of Art and Literature, Cambridge (MA), MIT Press, 2012, chap. 1, p. 27-74.

35. David Nye, American Technological Sublime, Cambridge (MA), MIT Press, 1994; Leo MarX, The Machine in the Garden. Technology and the Pastoral Ideal in America [1964], Oxford, Oxford University Press, 2000. 
La première couverture du magazine Life, créé par Henry Luce en 1936, représente justement un barrage, le Fort Peck Dam photographié par Margaret Bourke-White, alors connue notamment pour ses images d'usines et d'objets industriels ${ }^{36}$. Bourke-White avait déjà beaucoup contribué à diffuser une image positive de la grande industrie américaine, en particulier par sa collaboration avec le magazine Fortune, un hebdomadaire illustré consacré au commerce et à l'industrie, fondé lui aussi par Henry Luce en 1930. Le barrage, dont la construction fait partie de la politique de grands travaux conduite dans le cadre du New Deal, sous l'administration Roosevelt, est ici mis en scène de manière à en souligner le gigantisme. L'exploit technique qu'il représente est mis en contraste avec la petitesse de la figure humaine, qui en donne d'échelle. L'énorme masse du barrage, telle une falaise, convoque l'image de canyons ou de certains lieux emblématiques comme le parc du Yosemite, s'inscrivant ainsi dans l'imaginaire de la conquête de l'ouest et dans l'esthétique du sublime qui caractérise la tradition du paysage américain depuis le $\mathrm{XIX}^{\mathrm{e}}$ siècle ${ }^{37}$.

Le sublime industriel participe donc d'une stratégie, à laquelle les dirigeants d'entreprises et les industriels eux-mêmes contribuent, de naturalisation de la technologie et de nationalisation de l'idée de modernité (jusqu'alors plutôt associée à la culture européenne, notamment dans le champ artistique $\left.{ }^{38}\right)$. James S. Miller montre aussi comment Fortune a contribué à l'invention d'un "folklore industriel » légitimant le capitalisme industriel comme résultat d'un esprit d'entreprise authentiquement américain, vernaculaire et également partagé entre le peuple et les élites ${ }^{39}$. Alors que la glorification de l'industrie moderne semblait entrer en contradiction avec les courants artistiques régionalistes, alors très populaires aux États-Unis, le cas de Fortune démontre que la valorisation du vernaculaire et de traits "primitifs » américains sert à formuler une réconciliation entre le passé et le présent, à inscrire le développement technologique moderne dans une tradition ancienne.

36. Life, $\mathrm{n}^{\circ}$ 1, 23 novembre 1936, couverture en ligne : http://time.com/4570265/80th-anniversary-life-magazine/ (consulté le 14 mars 2019).

37. Anthony F. Arrigo, Imagining Hoover Dam: the Making of a Cultural Icon, Reno, University of Nevada Press, 2014.

38. Terry Sмiтh, Making the Modern: Industry, Art, and Design in America, Londres et Chicago, University of Chicago Press, 1993.

39. James S. Miller, "White-collar Excavations: Fortune Magazine and the Invention of the Industrial Folk ", American Periodicals, vol. 13, 2003, p. 84-104. 
Suivant une lecture de l'histoire en termes de civilisations (plutôt que de nations ou de classes sociales par exemple), les photographies du barrage de Fort Peck font de celui-ci un monument, qui serait l'équivalent des édifices colossaux édifiés par les civilisations archaïques : un monument de la civilisation machiniste. Cette articulation entre modernisme et primitivisme se retrouve d'ailleurs chez les peintres précisionnistes américains, comme Charles Sheeler ou Charles Demuth, qui intitula une de ses toiles représentant des silos My Egypt (1927, Whitney Museum of American Art, New York).

\section{La France, " pays de la mesure "}

Du point de vue français, la démesure technologique américaine suscite à la fois crainte et fascination. Dans un numéro spécial de $V u$ (30 mai 1936) consacré aux thèses de l'économiste Jacques Duboin ${ }^{40}$, une photographie du barrage Hoover est reproduite en double page (Fig. IV, cahier couleur). L'intervention des graphistes (Marc Réal et Alexandre Liberman) consiste à souligner l'extrême petitesse de l'homme confronté à la masse titanesque du barrage. Le graphique en partie droite met l'accent sur l'augmentation exponentielle de la production industrielle depuis les années 1920, tout en réitérant, dans la légende, la hantise d'une perte de contrôle face aux machines : " la machine aura-t-elle sa revanche ? L'homme perdra-t-il la maîtrise de son œuvre? ».

Face aux deux géants technologiques que représentent les États-Unis et l'URSS, journalistes et hommes politiques n'ont de cesse de présenter la France des années 1930 comme le "pays de la mesure ». C'est ainsi que titre $V u$, à l'occasion d'un numéro sur conséquences de la crise dans l'Hexagone $^{41}$. C'est également l'idéal qui transparaît dans l'un des recueils de photographies les plus diffusés dans les années 1930 : La France travaille de François Kollar (publié par fascicules de 1931 à 1934). Si par endroits les images de Kollar perpétuent certains codes de la photographie industrielle

40. Jacques Duboin est partisan d'une économie distributive fondée sur une accélération de la mécanisation et une réduction du temps de travail. VoirOlivier DARD, "Sortir de la crise par la technique et par la science ? Les réponses de l'abondancisme de Jacques Duboin ", L'Homme et la société, $\mathrm{n}^{\circ}$ 195-196, 2015/1, p. 127-148. Le numéro de Vu est publié peu après la victoire du Front populaire aux élections législatives.

41. $V u, n^{\circ} 220,1^{\text {er }}$ juin 1932. 
dans laquelle les ouvriers posent à côté de gigantesques machines, le photographe refuse généralement de s'inscrire dans la rhétorique de la démesure. La plupart des photographies montrent les ouvriers en plan rapproché, mettant l'accent sur leur l'habileté manuelle et le contrôle qu'ils exercent sur leur outil de travail. En somme, le travail de Kollar exemplifie l'attitude photographique dite humaniste, dans le sens où il s'oppose à la dépersonnalisation et la disproportion que nous avions identifiées comme les figures iconiques de la « crise de civilisation ».

Trois ans après Scènes de la vie future, Georges Duhamel publie une conférence intitulée L'Humaniste et l'automate, illustrée par des photographies de Jean Roubier ${ }^{42}$. Il y défend, contre les excès de la rationalisation technique, la transmission des savoirs manuels et la connaissance intuitive que peut avoir un praticien de son outil de travail, qui suppose de s'inscrire dans le temps long de l'apprentissage. Cet idéal " humaniste » de la technique qui caractérise le recueil de Kollar traverse ainsi les formulations visuelles et verbales du rapport homme-machine en France, avant de devenir un leitmotiv de la propagande du Front populaire.

L'Exposition internationale des «Arts et des Techniques appliqués à la Vie moderne ", en 1937, pourrait être interprétée globalement comme une tentative de la part du Front populaire de présenter la France comme le pays de la mesure, face aux démonstrations de puissance des pays totalitaires. Comme l'a montré Shanny Peer, l'image de la nation véhiculée par l'exposition serait celle d'une économie équilibrée, dans laquelle l'industrie ne prendrait pas le dessus sur l'agriculture ${ }^{43}$. Contrairement à certaines lectures rétrospectives qui voudraient y voir les sources de l'idéologie vichyste du « retour à la terre ", il faut comprendre l'Exposition de 1937 comme un effort de la part du gouvernement pour afficher l'image d'une modernisation mesurée, une position d'équilibre entre le productivisme des communistes et la hantise d'un machinisme excessif ${ }^{44}$.

42. Céline Glatard, "Jean Roubier et Georges Duhamel ", Études photographiques, n 33, automne 2015, en ligne : https://journals.openedition.org/etudesphotographiques/3557 (consulté le 31 octobre 2018).

43. Shanny Peer, France on Display: Peasants, Provincials, and Folklore in the 1937 Paris World's Fair, Albany, State University of New York Press, 1998, p. 14-20 ; Gérard NAMER, "Les imaginaires dans l'exposition de 1937 ", Cahiers internationaux de sociologie, vol. LXX, janvier-juin 1981, p. 35-62. 44. Romy Golan, "La possibilité d'un photomural socialiste ", in Maria Stavrinaki et Maddalena Carli (dir.), Artistes et partis : Esthétique et poltique (1900-1945), Dijon, Presses du réel, 2012, p. 231-251. 
François Kollar est très présent dans l'exposition. L'une de ses photographies est utilisée dans un photomontage monumental intitulé Travailler, réalisé par Fernand Léger pour le Pavillon des Temps Nouveaux. Conçu comme une vitrine pour l'activité des CIAM (Congrès Internationaux d'Architecture Moderne), le Pavillon déploie un propos didactique en faveur d'un urbanisme moderne, dont les soubassements philosophiques reposent sur une foi dans les vertus de la technologie maitrisée au service du progrès humain. De ce point de vue, le photomontage de Fernand Léger fonctionne comme l'emblème d'un rapport harmonieux à la technique, associant la dimension prométhéenne du projet moderne à un souci de mesure "humaniste ». Selon Romy Golan, « le motif se lit comme une actualisation de l'Homme vitruvien de Léonard, qui continue à se tenir, imperturbable, à l'épicentre du monde mécanique et reste, en tant que tel, la mesure de toute chose ${ }^{45}$ ".

Les outils de l'iconologie, appliqués à la culture visuelle des années 1930, révèlent la façon dont certaines figures traversent les discours et les images, dessinant les contours d'une mythologie politique cohérente. À "la machine " (au singulier, selon une hypostase récurrente dans les discours) répond le rouage comme allégorie visuelle du mouvement incontrôlé de l'histoire, dont on annonce alors l'issue fatale. Au topos verbal de la démesure correspondent autant d'images dans lesquelles les rapports de proportion fonctionnent comme éléments signifiants. Face à l'effondrement annoncé de la civilisation moderne, l'iconographie du déclin est à analyser pour sa valeur prospective et mobilisatrice. Fonctionnant sur le registre de l'anticipation, volontiers hyperbolique, le topos du déclin civilisationnel s'apparente à un "mythe social " au sens de Georges Sorel ${ }^{46}$, mais un mythe dont la portée politique est éminemment ambiguë. On voit bien en revanche comment, en fonction des différents contextes nationaux, s'opère une inflexion singulière du mythe : hommes-machines du fascisme et du nazisme, sublime technologique américain et soviétique, vision humaniste de la technique dans la France du Front populaire. Les résurgences de cette

45. Ibid., p. 251. Sur les réactualisations de l'homme de Vitruve, voir Giovanni Lista, Da Leonardo a Boccioni: l'Uomo vitruviano e l'arte moderna, Mudima, Milan, 2012.

46. Georges Sorel, Réflexions sur la violence (1908), Montreuil/Genève, Entremonde, 2013 ; Willy GianinazZI, "Images mentales et mythe social ", Mil neuf-cent. Revue d'histoire intellectuelle, $\mathrm{n}^{\circ} 28$, 2010, p. 155-172 ; Éric Michaud, "Le mythe social ou l'efficacité de l'image sans images ", Mil neuf-cent. Revue d'histoire intellectuelle, $\mathrm{n}^{\circ} 28,2010$, p. 173-183. 
mythologie sont également nombreuses dans l'après-guerre, en particulier dans le contexte de la menace nucléaire, qui réactualise la hantise de la démesure et l'idée d'une " obsolescence de l'homme ${ }^{47}$ ». Elle a aussi nourri une grande partie de l'imaginaire de la science-fiction, et trouve peut-être aujourd'hui une nouvelle actualité à l'heure de l'urgence écologique.

\section{L'auteur}

Max Bonhomme est doctorant en histoire de l'art à l'Université Paris-Nanterre, Labex Arts- $\mathrm{H} 2 \mathrm{H}$, sous la direction de Christian Joschke et Rémi Labrusse. Sa thèse porte sur les usages politiques du photomontage en France, de 1925 à 1945. Dans l'optique d'une histoire graphique de la presse, ses recherches portent sur le graphisme militant, l'iconographie politique et l'esthétique de la mise en page. Il a participé au projet de recherche « Photographie, arme de classe » et a publié dans le catalogue de l'exposition du même nom (Centre Pompidou, 2018). Contact : maxbonhomme@parisnanterre.fr

47. Günther Anders, L'Obsolescence de l'homme : sur l'àme à l'époque de la deuxième révolution industrielle (1956), trad. Christophe David, Paris, Ivrea, 2002. 